\title{
On the exact discretization of Schrödinger equation
}

\author{
Chih-Lung Chou \\ Department of Physics, Chung Yuan Christian University, Taoyuan 320314, Taiwan
}

\begin{abstract}
We show that the exact discrete analogue of Schröodinger equation can be derived naturally from the Hamiltonian operator of a Schrödinger field theory by using the discrete Fourier transform that transforms the operator from momentum representation into position representation. The standard central difference equation that is often used as the discretized Schrödinger equation actually describes a different theory since it is derived from a different Hamiltonian operator. The commutator relation between the position and momentum operators in discrete space is also derived and found to be different from the conventional commutator relation in continuous space. A comparison between the two discretization formulas is made by numerically studying the transmission probability for a wave packet passing through a square potential barrier in one dimensional space. Both discretization formulas are shown to give sensible and accurate numerical results as compared to theoretical calculation, though it takes more computation time when using the exact discretization formula. The average wave number $k_{0}$ of the incident wave packet must satisfy $\left|k_{0} \ell\right|<0.35$, where $\ell$ is the lattice spacing in position space, in order to obtain an accurate numerical result by using the standard central difference formula.
\end{abstract}

Keywords: Exact discretization, Schrodinger equation, commutator relation

Email address: ciwcielioong@gmail.com (Chih-Lung Chou)

Preprint submitted to Elsevier

August 11, 2020 


\section{Introduction}

In quantum mechanics the time-dependent Schrödinger equation is a second order linear partial differential equation

$$
i \hbar \frac{\partial}{\partial t} \Psi(x, t)=-\frac{\hbar^{2}}{2 M} \nabla^{2} \Psi(x, t)+U(x) \Psi(x, t),
$$

where the single-particle wavefunction $\Psi(x, t)$ is a function of position $x$ and time $t, U(x)$ denotes the potential energy, and $M$ is particle's mass. Though the Schrodinger equation has a specific form in terms of continuous variables $x$ and $t$, there are different discrete analogues of the equation [1, 2, 3]. For example, by using the standard central difference formula for the Laplace operator

$$
\nabla^{2} \Psi \rightarrow \sum_{a=1}^{3} \frac{1}{\ell^{2}}\{\Psi(x+\hat{a} \ell, t)+\Psi(x-\hat{a} \ell, t)-2 \Psi(x, t)\},
$$

a possible discretization of Eq.(1) is

$$
i \hbar \frac{\partial \Psi(x, t)}{\partial t}=-\frac{\hbar^{2}}{2 M \ell^{2}} \sum_{a=1}^{3}\{\Psi(x+\hat{a} \ell, t)+\Psi(x-\hat{a} \ell, t)-2 \Psi(x, t)\}+U(x) \Psi(x, t) .
$$

Here $\Psi(x, t)$ is defined only on the discrete position $x=\ell\left(\hat{1} n_{1}+\hat{2} n_{2}+\hat{3} n_{3}\right)$

${ }_{5}\left(n_{1}, n_{2}, n_{3}\right.$ are integers $), \hat{a}(a=1,2,3)$ denote the unit base vectors of the Cartesian coordinate system, and $\ell$ is the lattice spacing between the nearestneighboring spatial sites. Eq. (33) cannot be thought as an exact discrete analog of Eq.(1) since it has different dispersion relation $\varepsilon(k)$ than that in the continuous Schrödinger equation with zero-potential $U(x)=02$. In other words, the theory described by (3) is different from the theory described by the exact discrete analogue of the Schrödinger equation since both theories do have different Hamiltonian operators in discrete space. They are equal to each other only in the zero-spacing limit $\ell \rightarrow 0$.

An exact discretization of Schrodinger equation in one-dimensional space 
has been derived directly from the continuous Schrodinger equation [3]

$$
i \hbar \frac{d \Psi(x, t)}{d t}=\frac{\hbar^{2}}{M \ell^{2}}\left\{\frac{\pi^{2}}{6} \Psi(x, t)+\sum_{\substack{m=-\infty \\ m \neq 0}}^{\infty} \frac{(-1)^{m}}{m^{2}} \Psi(x-m \ell, t)\right\}+U(x) \Psi(x, t) .
$$

Different from the standard central difference formula, the exact discretized

Schrödinger equation has difference of integer order that is represented by infinite series, and a long-range interaction is suggested in the discretized equation.

In this paper, we show that a natural way for the derivation of the exact discretized Schrödinger equation is from the Hamiltonian operator of the Schrödinger field theory. It is known that the continuous Schrödinger equation that describes the time evolution of wavefunction can be derived from the following equation

$$
i \hbar \frac{\partial}{\partial t}|\Psi\rangle=H|\Psi\rangle
$$

where the Hamiltonian operator $H$ plays the role of the generator of time evolution. The so-called wavefunction $\Psi(x, t)$ is the inner product between the position eigenket $|x\rangle$ and the quantum state $|\Psi\rangle, \Psi(x, t)=\langle x \mid \Psi\rangle$. Once the Hamiltonian operator is given, by taking the inner product on both sides of (5), the Schrödinger equation in (1) is obtained. Following the same line of thought, the exact discretized Schrödinger equation could also be derived from the Hamiltonian operator of the quantum system in discrete space. For example, consider a Schrödinger field theory with the Lagrangian density [4]

$$
\begin{aligned}
\mathcal{L} & =\frac{i \hbar}{2}\left\{\Psi^{*}(x, t) \frac{\partial}{\partial t} \Psi(x, t)-\left[\frac{\partial}{\partial t} \Psi^{*}(x, t)\right] \Psi(x, t)\right\}-\frac{\hbar^{2}}{2 m} \nabla \Psi^{*}(x, t) \cdot \nabla \Psi(x, t) \\
& +U(x) \Psi^{*}(x, t) \Psi(x, t)
\end{aligned}
$$

the continuous Schrödinger equation is derived as the equation of motion in the theory. Without the self interaction term (ie., $U(x)=0$ ), after the second quantization of the free theory in discrete space, the free Hamiltonian operator 
is diagonal in the discrete momentum space

$$
H_{0}=\sum_{k} \varepsilon_{k} a_{k}^{\dagger} a_{k}
$$

where $\varepsilon_{k}=\hbar^{2} k^{2} / 2 M$ denotes the energy for a free particle with momentum $\hbar k$, and $a_{k}^{\dagger}$ and $a_{k}$ are the creation and the annihilation operator that satisfy the quantization relation

$$
\left[a_{k}, a_{k^{\prime}}^{\dagger}\right]=\delta_{k k^{\prime}}
$$

Here the momentum vector $k$ takes only discrete values. Once the free Hamiltonian operator $H_{0}$ is obtained in momentum representation, it is straightforward to rewrite $H_{0}$ in position representation by using discrete Fourier transforms. Obviously, $H_{0}$ will not be diagonal in position representation due to Heisenberg's uncertainty relation, thus a spatially localized particle has a chance to hop to other locations instead of staying at the same position in the free theory. With the presence of the self interaction $U(x)$, the Hamiltonian operator becomes

$$
H=H_{0}+\sum_{x} U(x) a_{x}^{\dagger} a_{x} .
$$

Here the potential energy part of $H$ is diagonal in position representation. Once the Hamiltonian operator $H$ in position representation is obtained, the exact discrete analogue of Schrödinger equation can be derived from (5) by

$$
i \hbar \frac{\partial}{\partial t}\langle x \mid \Psi\rangle=\langle x|H| \Psi\rangle
$$

In the next section we show that the exact discretization of Schrodinger equation can be obtained by transforming $H_{0}$ from momentum representation into position representation. In the third section, we discuss the discrete version 20 of the commutator relation $[\hat{X}, \hat{P}]$ between position operator $\hat{X}$ and momentum operator $\hat{P}$. Next we compare the exact discretized Schrödinger equation with the standard central difference formula by numerically studying the problem of a wave packet passing through a potential barrier. In the last section we give our conclusion. 


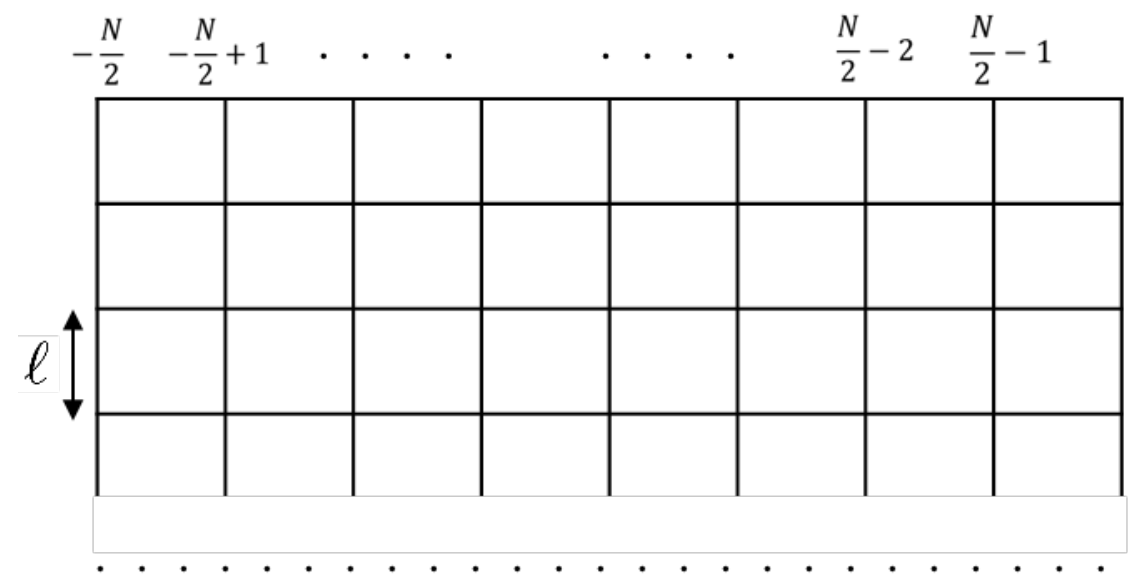

Figure 1: The three-dimensional discrete position space with a square lattice structure. The dimension in each direction is $L=N \ell$.

\section{Derivation of the exact discretized Schrodinger equation}

Consider a discrete position space with a square lattice structure that is described by

$$
x=\ell\left(\hat{1} n_{1}+\hat{2} n_{2}+\hat{3} n_{3}\right), \quad-\frac{N}{2} \leq n_{1}, n_{2}, n_{3} \leq \frac{N}{2}-1 .
$$

Here $x$ denotes the position of the spatial lattice sites, $n_{1}, n_{2}$, and $n_{3}$ are integers, $\hat{a}(a=1,2,3)$ denote the unit base vectors of the Cartesian coordinate system, $\ell$ is the lattice spacing, and $N$ is a large even number. As shown in Fig 1, the three-dimensional discrete position space can be viewed as a cube with side length $L=N \ell$ and total volume $L^{3}$. Given a free Schrödinger field theory in the discrete space with Hamiltonian $H_{0}$, the operator $H_{0}$ is diagonal in momentum space and has the form as described in (7). The diagonal form of $H_{0}$ in momentum space is indeed a direct consequence of the translational symmetry in position space in the free theory. In the free theory, a normalized single particle state with momentum $\mathrm{k}$ is

$$
|k\rangle=a_{k}^{\dagger}|0\rangle,
$$


where $|0\rangle$ denotes the vacuum state, and the momentum $k$ takes only discrete values

$$
k=\frac{2 \pi}{L}\left(m_{1}, m_{2}, m_{3}\right), \quad-\frac{N}{2} \leq m_{1}, m_{2}, m_{3} \leq \frac{N}{2}-1 .
$$

The integers $m_{1}, m_{2}$ and $m_{3}$ range from $-N / 2$ to $N / 2-1$. The discrete momentum space also has a square lattice structure but with lattice spacing $2 \pi / L$.

To transform Hamiltonian from momentum representation into position representation, we define the field operator $a_{x}$ and its hermitian conjugate $a_{x}^{\dagger}$ as the discrete Fourier transforms of $a_{k}$ and $a_{k}^{\dagger}$

$$
\begin{aligned}
& a_{x}=\frac{1}{\sqrt{N^{3}}} \sum_{k} e^{i \vec{k} \cdot \vec{x}} a_{k}, \\
& a_{x}^{\dagger}=\frac{1}{\sqrt{N^{3}}} \sum_{k} e^{-i \vec{k} \cdot \vec{x}} a_{k}^{\dagger} .
\end{aligned}
$$

Here the discrete momentum $k$ is defined in (13), and $x$ denotes the position of lattice sites in the three-dimensional discrete position space. From the quantization relation in (8), the field operators $a_{x}$ and $a_{x}^{\dagger}$ are shown to satisfy the commutator relation

$$
\left[a_{x}, a_{x^{\prime}}^{\dagger}\right]=\delta_{x x^{\prime}}
$$

The commutator relation in 16 thus allows us to define the position eigenkets as

$$
|x\rangle=a_{x}^{\dagger}|0\rangle .
$$

The position eigenkets are normalized states, like the momentum states $|k\rangle$, can also be single-particle states in the Schrödinger field theory in the discrete space. From (14) and 15, $a_{k}$ and $a_{k}^{\dagger}$ can be written as linear combinations of $a_{x}$ and $a_{x}^{\dagger}$

$$
\begin{aligned}
& a_{k}=\frac{1}{\sqrt{N^{3}}} \sum_{x} e^{-i \vec{k} \cdot \vec{x}} a_{x}, \\
& a_{k}^{\dagger}=\frac{1}{\sqrt{N^{3}}} \sum_{x} e^{i \vec{k} \cdot \vec{x}} a_{x}^{\dagger} .
\end{aligned}
$$


Taking Eq.s 18 and 19 into $H_{0}$ in $(7)$, we have

$$
H_{0}=\frac{1}{N^{3}} \sum_{x} \sum_{k} \varepsilon_{k} a_{x}^{\dagger} a_{x}+\frac{1}{N^{3}} \sum_{\substack{x, x^{\prime} \\ x \neq x^{\prime}}} \sum_{k} \varepsilon_{k} e^{i \vec{k} \cdot\left(\vec{x}-\vec{x}^{\prime}\right)} a_{x}^{\dagger} a_{x^{\prime}} .
$$

The form of $H_{0}$ in 20 can be simplified further if the single-particle energy $\varepsilon_{k}$ is known. In the Schrödinger field theory, $\varepsilon_{k}=\hbar^{2} k^{2} / 2 M$, the sum $\left(1 / N^{3}\right) \sum_{k} \varepsilon_{k}$ in 20 is found to be

$$
\begin{aligned}
\frac{\hbar^{2}}{2 M N^{3}} \sum_{k} k^{2} & =\frac{3 \hbar^{2}}{2 M N}\left(\frac{2 \pi}{L}\right)^{2} \sum_{n=-N / 2}^{N / 2-1} n^{2}=\frac{\hbar^{2} \pi^{2}}{2 M \ell^{2}}\left(1+\frac{2}{N^{2}}\right) \\
& \longrightarrow \frac{\hbar^{2} \pi^{2}}{2 M \ell^{2}}, \quad \text { as } N \rightarrow \infty .
\end{aligned}
$$

Using the fact that

$$
\sum_{k_{a}} e^{i k_{a}\left(n-n^{\prime}\right) \ell}=N \delta_{n n^{\prime}}, \quad a=1,2,3 .
$$

where $k_{a}$ is the $a$-th component of wave vector $k, n$ and $n^{\prime}$ are integers. The other sum $\left(1 / 2 M N^{3}\right) \sum_{k} k^{2} e^{i \vec{k} \cdot\left(\vec{x}-\vec{x}^{\prime}\right)}$ in 20 with nonzero spatial separation $\left(\vec{x}-\vec{x}^{\prime}\right)$ is found be nonzero only when $\left(\vec{x}-\vec{x}^{\prime}\right)$ lies in either direction $\hat{1}, \hat{2}$, or $\hat{3}$. Let assume that $\vec{x}-\vec{x}^{\prime}=\hat{1} m \ell(m \neq 0)$, then the sum is

$$
\begin{aligned}
& \frac{1}{2 M N^{3}} \sum_{k} k^{2} e^{i \vec{k} \cdot\left(\vec{x}-\vec{x}^{\prime}\right)}=\frac{2 \pi^{2}}{M N L^{2}} \sum_{n=-N / 2}^{N / 2-1} n^{2}\left(e^{i 2 \pi m / N}\right)^{n} \\
& =\frac{2 \pi^{2}}{M N^{3} \ell^{2}}\left\{(-1)^{m} \frac{N^{2}}{4}+2 \sum_{n=1}^{N / 2-1} n^{2} \cos (2 \pi m n / N)\right\} \\
& =\frac{(-1)^{m} \pi^{2}}{M N^{2} \ell^{2} \sin ^{2}(m \pi / N)}, \quad m \neq 0 .
\end{aligned}
$$

In the $N \rightarrow \infty$ limit, when $\vec{x}=\vec{x}^{\prime}+\hat{a} m \ell(a=1,2,3)$, the sum in 23) becomes

$$
\frac{1}{2 M N^{3}} \sum_{k} k^{2} e^{i \vec{k} \cdot\left(\vec{x}-\vec{x}^{\prime}\right)} \longrightarrow \frac{(-1)^{m}}{M \ell^{2} m^{2}} .
$$

Finally, from Eq.s 20, 21, 24, the free Hamiltonian operator in the large $N$ limit in position representation is

$$
H_{0}=\frac{\hbar^{2}}{M \ell^{2}}\left\{\frac{\pi^{2}}{2} \mathbb{1}+\sum_{x} \sum_{\substack{m=-\infty \\ m \neq 0}}^{\infty} \sum_{a=1}^{3} \frac{(-1)^{m}}{m^{2}} a_{x+\hat{a} m \ell}^{\dagger} a_{x}\right\} .
$$


Here $\mathbb{1}=\sum_{x} a_{x}^{\dagger} a_{x}$ denotes the identity operator. $H_{0}$ is non-diagonal in position representation with the presence of the hopping interaction $a_{x+\hat{a} m \ell}^{\dagger} a_{x}$. The hopping interaction terms are responsible for the position-momentum uncertainty relation $\Delta x \Delta p \geq \hbar / 2$. With the hopping terms, a spatially localized particle can hop to other places rather than just staying at the same location. Turning on potential energy $U(x)$, the Hamiltonian operator for the Schrödinger field theory is

$$
H=\frac{\hbar^{2}}{M \ell^{2}}\left\{\frac{\pi^{2}}{2} \mathbb{1}+\sum_{x} \sum_{\substack{m=-\infty \\ m \neq 0}}^{\infty} \sum_{a=1}^{3} \frac{(-1)^{m}}{m^{2}} a_{x+\hat{a} m \ell}^{\dagger} a_{x}\right\}+\sum_{x} U(x) a_{x}^{\dagger} a_{x} .
$$

Similarly, in two dimensional and one dimensional discrete space, the Hamiltonian operators for the corresponding Schrödinger field theories are

$$
\begin{aligned}
H^{2 d i m} & =\frac{\hbar^{2}}{M \ell^{2}}\left\{\frac{\pi^{2}}{3} \mathbb{1}+\sum_{x} \sum_{\substack{m=-\infty \\
m \neq 0}}^{\infty} \sum_{a=1}^{2} \frac{(-1)^{m}}{m^{2}} a_{x+\hat{a} m \ell}^{\dagger} a_{x}\right\}+\sum_{x} U(x) a_{x}^{\dagger} a_{x}, \\
H^{1 d i m} & =\frac{\hbar^{2}}{M \ell^{2}}\left\{\frac{\pi^{2}}{6} \mathbb{1}+\sum_{x} \sum_{\substack{m=-\infty \\
m \neq 0}}^{\infty} \frac{(-1)^{m}}{m^{2}} a_{x+m \ell}^{\dagger} a_{x}\right\}+\sum_{x} U(x) a_{x}^{\dagger} a_{x} .
\end{aligned}
$$

The exact discrete analog of Schrodinger equation is derived as follows. Let's take the one-dimensional Schrödinger theory as an example. Consider a singleparticle quantum state $|\Psi\rangle$ in position representation

$$
|\Psi\rangle=\sum_{x} \Psi(x, t) a_{x}^{\dagger}|0\rangle=\sum_{x} \Psi(x, t)|x\rangle
$$

From (5) and (28), it has

$$
\begin{aligned}
& \sum_{x} i \hbar \frac{\partial \Psi(x, t)}{\partial t}|x\rangle \\
= & \frac{\hbar^{2}}{M \ell^{2}}\left\{\frac{\pi^{2}}{6} \sum_{x} \Psi(x, t)|x\rangle+\sum_{x} \sum_{\substack{m=-\infty \\
m \neq 0}}^{\infty} \frac{(-1)^{m}}{m^{2}} \Psi(x, t)|x+m \ell\rangle\right\}+\sum_{x} U(x) \Psi(x, t)|x\rangle .
\end{aligned}
$$


Comparing the coefficient of $|x\rangle$ on both sides of the equation (30), we get

$$
i \hbar \frac{\partial \Psi(x, t)}{\partial t}=\frac{\hbar^{2}}{M \ell^{2}}\left\{\frac{\pi^{2}}{6} \Psi(x, t)+\sum_{\substack{m=-\infty \\ m \neq 0}}^{\infty} \frac{(-1)^{m}}{m^{2}} \Psi(x-m \ell, t)\right\}+U(x) \Psi(x, t) .
$$

The result in (31) is indeed the exact discrete analogue of Schrödinger equation given in (3). Using the identity

$$
\sum_{m=1}^{\infty}(-1)^{m} / m^{2}=-\frac{\pi^{2}}{12}
$$

the equation in 31 can be further written as

$$
\begin{aligned}
& i \hbar \frac{\partial \Psi(x, t)}{\partial t} \\
= & \frac{\hbar^{2}}{M} \sum_{m=1}^{\infty}\left\{\frac{(-1)^{m}}{m^{2} \ell^{2}}[\Psi(x+m \ell, t)+\Psi(x-m \ell, t)-2 \Psi(x, t)]\right\}+U(x) \Psi(x, t) .
\end{aligned}
$$

Eq. (33) shows that the second order differential operation $\partial^{2} \Psi(x, t) / \partial x^{2}$ can be replaced by the infinite difference

$$
\frac{\partial^{2} \Psi(x, t)}{\partial x^{2}} \rightarrow-2 \sum_{m=1}^{\infty} \frac{(-1)^{m}}{m^{2} \ell^{2}}[\Psi(x+m \ell, t)+\Psi(x-m \ell, t)-2 \Psi(x, t)],
$$

in the discrete version of quantum mechanics. In two $(D=2)$ or three $(D=3)$ dimensional space, the exact discretized Schrödinger equation is

$$
\begin{aligned}
& i \hbar \frac{\partial \Psi(x, t)}{\partial t} \\
= & \frac{\hbar^{2}}{M} \sum_{a=1}^{D} \sum_{m=1}^{\infty}\left\{\frac{(-1)^{m}}{m^{2} \ell^{2}}[\Psi(x+\hat{a} m \ell, t)+\Psi(x-\hat{a} m \ell, t)-2 \Psi(x, t)]\right\}+U(x) \Psi(x, t) .
\end{aligned}
$$

Now we know that Eq. 35 is an exact analogue of the continuous Schrödinger equation since it is derived directly from the Hamiltonian operator of the theory in discrete space. The standard central difference equation given in (3) is only an approximate. In fact, Eq.(3) actually describes another quantum theory that 
has the Hamiltonian operator

$$
H=H_{0}+\sum_{x} U(x) a_{x}^{\dagger} a_{x}
$$

with

$$
\begin{aligned}
H_{0} & =\frac{3 \hbar^{2}}{M \ell^{2}}\left\{\mathbb{1}-\frac{1}{6} \sum_{x} \sum_{b=1}^{3}\left(a_{x+\hat{b} \ell}^{\dagger} a_{x}+a_{x-\hat{b} \ell}^{\dagger} a_{x}\right)\right\} \\
& =\frac{3 \hbar^{2}}{M \ell^{2}} \sum_{k}\left[1-\frac{1}{3} \sum_{b=1}^{3} \cos \left(k_{b} \ell\right)\right] a_{k}^{\dagger} a_{k} .
\end{aligned}
$$

Obviously, the Hamiltonian operator given in $(36,37,38)$ is different from the Hamiltonian operator given in (26). The dispersion relation $\varepsilon_{k}$ in (38)

$$
\varepsilon_{k}=\frac{3 \hbar^{2}}{M \ell^{2}}\left(1-\frac{1}{3} \sum_{b=1}^{3} \cos \left(k_{b} \ell\right)\right),
$$

is effectively the same dispersion relation given in 2. It differs from the free particle energy $\hbar^{2} k^{2} / 2 M$ by less than one percent if $\left|k_{b} \ell\right|<0.35$. The two

so Hamiltonian operators indeed describe different quantum theories in discrete space. When $\left|k_{b} \ell\right|<0.35$ is satisfied, the theory described by the Hamiltonian in $(38)$ could be a good approximation of the theory that is described by the Hamiltonian in (7). They are equal to each other only in the $\ell \rightarrow 0$ limit.

\section{Discrete analogue of canonical quantization relation}

In Schrodinger field theory in discrete space, the position and momentum operators in $\hat{b}$-direction $(b=1,2,3)$ are defined as

$$
\begin{aligned}
& \hat{P}_{b}=\sum_{k} \hbar k_{b} a_{k}^{\dagger} a_{k}, \quad b=1,2,3 . \\
& \hat{X}_{b}=\sum_{x} x_{b} a_{x}^{\dagger} a_{x}, \quad b=1,2,3 .
\end{aligned}
$$

With the definition of momentum operator, a position eigenstate $\left|x_{0}\right\rangle$ will have a non-zero mean value of $\hat{P}_{b}$

$$
\left\langle x_{0}\left|\hat{P}_{b}\right| x_{0}\right\rangle=-\frac{\hbar \pi}{L}
$$


It is due to the asymmetric range of $k_{b}$

$$
k_{b}=\frac{2 \pi}{L} n_{b}, \quad n_{b}=\frac{-N}{2}, \frac{-N}{2}+1, \ldots, \frac{N}{2}-1 .
$$

The non-zero mean value can be removed either by removing $k_{b}=-\pi / \ell$ from the allowable values of $k_{b}$ or by taking the limit $L \rightarrow \infty$. Similarly, a momentum state $\left|k_{0}\right\rangle$ also has a non-zero mean $\left\langle k_{0}\left|\hat{X}_{b}\right| k_{0}\right\rangle=-\ell / 2$ due to the asymmetry of the range of $x_{b}$. It can also be removed either by removing $x_{b}=-L / 2$ from the allowable eigenvalues of $\hat{X}_{b}$ or by taking the $\ell \rightarrow 0$ limit. In position representation, the momentum operator becomes

$$
\begin{aligned}
\hat{P}_{b} & =\frac{\hbar}{N^{3}} \sum_{x, y} \sum_{k} k_{b} e^{i \vec{k} \cdot(\vec{x}-\vec{y})} a_{x}^{\dagger} a_{y} \\
& =\frac{\hbar}{N^{3}}\left\{\sum_{x} \sum_{k} k_{b} a_{x}^{\dagger} a_{x}+\sum_{k} \sum_{\substack{x, y \\
x \neq y}} k_{b} e^{i \vec{k} \cdot(\vec{x}-\vec{y})} a_{x}^{\dagger} a_{y}\right\} \\
& =-\frac{\hbar \pi}{L}\left\{\sum_{x} \sum_{m}(-1)^{m} a_{x+\hat{b} m \ell}^{\dagger} a_{x}+i \sum_{x} \sum_{m \neq 0}(-1)^{m} \cot \left(\frac{m \pi}{N}\right) a_{x+\hat{b} m \ell}^{\dagger} a_{x}\right\} .
\end{aligned}
$$

The first part of momentum operator in 44

$$
-\frac{\hbar \pi}{L} \sum_{x} \sum_{m}(-1)^{m} a_{x+\hat{b} m \ell^{\dagger}}^{a_{x}}
$$

is also due to the asymmetry of the range of $k_{b}$. It disappears if a symmetric momentum operator is defined

$$
\hat{P}_{b}=\sum_{\substack{k \\ k_{b} \neq-\pi / \ell}} \hbar k_{b} a_{k}^{\dagger} a_{k}, \quad b=1,2,3 .
$$

For spatially smooth wavefunction $\Psi(x, t)$ the first part of $\hat{P}_{b}$ has a negligible contribution to $\left\langle x\left|\hat{P}_{b}\right| \Psi\right\rangle$ since $(-1)^{m} \Psi(x-\hat{b} m \ell, t)$ oscillates extremely rapidly in space as $m$ increases

$$
\left\langle x\left|\frac{\hbar \pi}{L} \sum_{x^{\prime}} \sum_{m}(-1)^{m} a_{x^{\prime}+\hat{b} m \ell}^{\dagger} a_{x^{\prime}}\right| \Psi\right\rangle=\frac{\hbar \pi}{L} \sum_{m}(-1)^{m} \Psi(x-\hat{b} m \ell, t) \ll 1 .
$$


In the $\sqrt{N} \ell \rightarrow \infty$ limit

$$
\left|\left\langle x\left|\frac{\hbar \pi}{L} \sum_{x^{\prime}} \sum_{m}(-1)^{m} a_{x^{\prime}+\hat{b} m \ell}^{\dagger} a_{x^{\prime}}\right| \Psi\right\rangle\right| \leq \frac{\hbar \pi}{L} \sum_{m}|\Psi(x-\hat{b} m \ell, t)| \leq \frac{\hbar \pi}{\sqrt{N} \ell} \longrightarrow 0 .
$$

Here $\Psi(x, t)$ is assumed to be a normalized wavefunction, $\sum_{x}|\Psi(x, t)|^{2}=1$, so that $\sum_{m}|\Psi(x-\hat{b} m \ell, t)| \leq \sqrt{N}$. Therefore, in the $\sqrt{N} \ell \rightarrow \infty$ limit for any normalized quantum state $|\Psi\rangle$, the exact discrete analogue of the differential operation becomes $[3,5]$

$$
\frac{i}{\hbar}\left\langle x\left|\hat{P}_{b}\right| \Psi\right\rangle=\frac{\partial \Psi(x, t)}{\partial x_{b}} \Rightarrow \sum_{\substack{m=-\infty \\ m \neq 0}}^{\infty} \frac{(-1)^{m}}{m \ell} \Psi(x+\hat{b} m \ell, t) .
$$

The commutator relation between $\hat{X}_{a}$ and $\hat{P}_{b}$ is found in a similar way. From (40) and 41,

$$
\begin{aligned}
{\left[\hat{X}_{a}, \hat{P}_{b}\right] } & =\hbar \sum_{x} \sum_{k} x_{a} k_{b}\left[a_{x}^{\dagger} a_{x}, a_{k}^{\dagger} a_{k}\right] \\
& =\frac{\hbar}{N^{3}} \sum_{x, x^{\prime}} \sum_{k} k_{b}\left(x_{a}-x_{a}^{\prime}\right) e^{i \vec{k} \cdot\left(\vec{x}-\vec{x}^{\prime}\right)} a_{x}^{\dagger} a_{x^{\prime}} .
\end{aligned}
$$

The result in 50 is not zero only when $a=b$. Without loss of generality, let's consider the case that $a=b=1$. Using

$$
\sum_{k} k_{1} e^{i k_{1}\left(x_{1}-x_{1}^{\prime}\right)}=\frac{2 \pi}{L} \sum_{n=-N / 2}^{N / 2-1} n e^{i 2 \pi n m / N}=(-1)^{m+1} \frac{\pi}{\ell}\left(1+i \cot \left(\frac{\pi m}{N}\right)\right),
$$

where $k_{1}=2 \pi n / L$ and $x_{1}-x_{1}^{\prime}=m \ell \neq 0$, the commutator $\left[\hat{X}_{1}, \hat{P}_{1}\right]$ is

$$
\begin{aligned}
{\left[\hat{X}_{1}, \hat{P}_{1}\right] } & =-\hbar \sum_{x} \sum_{m \neq 0}(-1)^{m}\left(\frac{\pi m}{N}\right)\left(1+i \cot \left(\frac{\pi m}{N}\right)\right) a_{x}^{\dagger} a_{x-\hat{1} m \ell} . \\
& =i \hbar \sum_{x} a_{x}^{\dagger} a_{x}-\hbar \sum_{x} \sum_{m}(-1)^{m}\left(\frac{\pi m}{N}\right)\left(1+i \cot \left(\frac{\pi m}{N}\right)\right) a_{x}^{\dagger} a_{x-\hat{\imath} m \ell} .
\end{aligned}
$$

Here the integer $m$ ranges from $-N / 2 \leq\left(x_{1} / \ell-n\right) \leq N / 2-1$. From the above results, we get the commutator relation

$$
\left[\hat{X}_{a}, \hat{P}_{b}\right]=i \hbar \delta_{a b} \mathbb{1}-\hbar \delta_{a b} \sum_{x} \sum_{m}(-1)^{m}\left(\frac{\pi m}{N}\right)\left(1+i \cot \left(\frac{\pi m}{N}\right)\right) a_{x}^{\dagger} a_{x-\hat{a} m \ell} .
$$


35 The commutator relation shown in (53) differs from the conventional quantization relation $\left[\hat{X}_{a}, \hat{P}_{b}\right]=i \hbar \delta_{a b} \mathbb{1}$ in continuous space. Since for a Schrödinger theory that is defined on a discrete space with finite number of lattice sites (the total number of sites is $N^{3}$ ), all momentum eigenstates $|k\rangle$ and position eigenstates $|x\rangle$ are normalized states. This means that all $|k\rangle$ and $|x\rangle$ eigen-

40 kets can be used as the single-particle quantum states in the theory if relativistic energy is allowed (For highly localized state $|\Psi\rangle=|x\rangle$, the particle state has energy $\left.\langle\Psi|H| \Psi\rangle=\pi^{2} \hbar^{2} / 2 M \ell^{2} \gg M c^{2}\right)$. Thus, the relations that $\left\langle k\left|\left[\hat{X}_{a}, \hat{P}_{b}\right]\right| k\right\rangle=0=\left\langle x\left|\left[\hat{X}_{a}, \hat{P}_{b}\right]\right| x\right\rangle$ help to explain why the conventional commutator relation $\left[\hat{X}_{a}, \hat{P}_{b}\right]=i \hbar \delta_{a b} \mathbb{1}$ cannot be correct in discrete space.

When the commutator acts upon a quantum state $|\psi\rangle=\sum_{x} \Psi(x, t)|x\rangle$, it gives

$\left\langle x\left|\left[\hat{X}_{a}, \hat{P}_{b}\right]\right| \Psi\right\rangle=\hbar \delta_{a b}\left\{i \Psi(x, t)-\sum_{m}(-1)^{m}\left(\frac{\pi m}{N}\right)\left(1+i \cot \left(\frac{\pi m}{N}\right)\right) \Psi(x+\hat{a} m \ell, t)\right\}$.

The infinite sum in (54) has extremely small contribution when the normalized wavefunction $\Psi(x, t)$ is a localized and relatively smooth function in position space. In the situation, $(-1)^{m}\left(\frac{\pi m}{N}\right)\left(1+i \cot \left(\frac{\pi m}{N}\right)\right) \Psi(x+\hat{a} m \ell, t)$ is not only a finite localized function but also oscillates very rapidly in space. So, in the limit that $N \gg 1$ and $\ell \rightarrow 0$,

$$
\sum_{m}(-1)^{m}\left(\frac{\pi m}{N}\right)\left(1+i \cot \left(\frac{\pi m}{N}\right)\right) \Psi(x+\hat{a} m \ell, t) \longrightarrow 0 .
$$

Thus in the continuous limit, for any quantum state $|\Psi\rangle$ with a localized and relatively smooth wavefunction, the conventional commutator relation holds

$$
\left\langle x\left|\left[\hat{X}_{a}, \hat{P}_{b}\right]\right| \Psi\right\rangle=i \hbar \delta_{a b} \Psi(x, t) .
$$

\section{4. The exact discretization versus the standard central difference equation}

The problems of a Gaussian wave packet passing through one or two potential barriers have been studied either theoretically or numerically [6, 7]. In the paper 
a comparison between the exact discrete analogue of Schrödinger equation and the standard central difference equation is made by numerically studying the transmission probability for a Gaussian wave packet passing through a square potential barrier in one dimensional space. We assume the initial wavefunction for a particle to be a Gaussian wave packet with its center located at $x_{0}$

$$
\Psi(x, t=0)=\left(\frac{1}{\sqrt{2 \pi} \sigma}\right)^{1 / 2} \exp \left[-\frac{\left(x-x_{0}\right)^{2}}{4 \sigma^{2}}+i k_{0}\left(x-x_{0}\right)\right]
$$

Here $\sigma$ is the standard deviation of particle's probability distribution at time $t=0$. The initial wave packet $\Psi(x, 0)$ actually consists of many plane waves with wave numbers $k$ around $k_{0}\left(k_{0}>0\right)$

$$
\Psi(x, 0)=\frac{1}{\sqrt{2 \pi}} \int d k \Phi(k) e^{i k\left(x-x_{0}\right)},
$$

where $\Phi(k)$ is

$$
\Phi(k)=\left(\frac{2 \sigma^{2}}{\pi}\right)^{1 / 4} \exp \left[-\sigma^{2}\left(k-k_{0}\right)^{2}\right] .
$$

Since $\Phi(k)$ is symmetric about $k_{0}$, the average energy for the wave packet is thus $E_{0}=\hbar^{2} k_{0}^{2} / 2 M$. The wavefunction at a later time is obtained by solving Schrödinger equation. Using the exact discretized Schrödinger equation in (35), the wave function $\Psi(x, t+\Delta t)$ at a later time $t+\Delta t$ can be obtained from the same wavefunction at the previous time $t$

$$
\begin{aligned}
\Psi(x, t+\Delta t) & =\Psi(x, t)-i \Delta \tau\left\{\sum_{m>0} \frac{(-1)^{m}}{m^{2}}[\Psi(x+m \ell, t)+\Psi(x-m \ell, t)\right. \\
& -2 \Psi(x, t)]+\eta(x) \Psi(x, t)\}+O\left((\Delta \tau)^{2}\right)
\end{aligned}
$$

where $\Delta \tau \equiv \hbar \Delta t /\left(M \ell^{2}\right)$ denotes the time step parameter in numerical calculation, and $\eta(x)$ is defined as

$$
\eta(x)=\frac{M \ell^{2} U(x)}{\hbar^{2}}=\frac{\left(k_{0} \ell\right)^{2} U(x)}{2 E_{0}} .
$$

As long as $\Delta \tau$ is small enough the numerical calculation based on 60 should give an accurate and sensible result, and does not depend on the lattice spacing $\ell$ directly. On the other hand, if the standard central difference equation is 
used in numerical calculation, then $\Psi(x, t+\Delta t)$ is merely determined by the wavefunction at the previous time $t$ at site $x$ and its nearest neighbors $x \pm \ell$

$$
\begin{aligned}
\Psi(x, t+\Delta t) & =\Psi(x, t)-i \Delta \tau\left\{\Psi(x, t)-\frac{1}{2}[\Psi(x+\ell, t)+\Psi(x-\ell, t)]\right. \\
& +\eta(x) \Psi(x, t)\}+O\left((\Delta \tau)^{2}\right) .
\end{aligned}
$$

Numerical calculation based on 62 needs not only the enough small value of $\Delta \tau$ but also the condition $\left|k_{0} \ell\right|<0.35$. Unlike the use of the exact discretization in $(60)$, a suitable choice of the spatial lattice spacing $\ell$ is important in getting a reasonable numerical result. The condition $\left|k_{0} \ell\right|<0.35$ guarantees that the theory described by the standard central difference equation is close to the conventional Schrödinger field theory. Actually, the discrepancy in free particle's energy between the two theories is less than one percent if $\left|k_{0} \ell\right|<0.35$ is satisfied. From the aspect of numerical calculation, the most important difference between the two discretization methods is the non-local nature in the exact discretization method. In $60, \Psi(x, t+\Delta t)$ is determined by the same wavefunction at all spatial lattice sites in the previous time moment $t$. It is very different from the standard central discretization in which $\Psi(x, t+\Delta t)$ is only determined by the wavefunction at the same and the nearest neighboring sites 60 in the previous time $t$. Thus a numerical calculation based on the exact discretization formula usually takes a much longer execution time than that based on the standard central difference equation.

Fig 2 shows the reflected and transmitted wave of an incident wave packet passing through a potential barrier that is located at the center of space with height $U$ and barrier width $W=10 \ell$. The space is one-dimensional with $N=$ 500 lattice sites and the lattice spacing is set as $\ell=1$. The average energy $E_{0}$ for the incident particle is chosen as $E_{0} / U=\pi^{2} / 8.0$ with $k_{0}=\pi / 6$, and the standard deviation $\sigma$ of the probability distribution of the initial wave packet is $\sigma=15 \ell$. By choosing the time step $\Delta \tau=0.001$, the numerical calculation based on 60 gives the transmission probability

$$
\mathrm{P}(\text { transmission })=0.654 \text {, }
$$




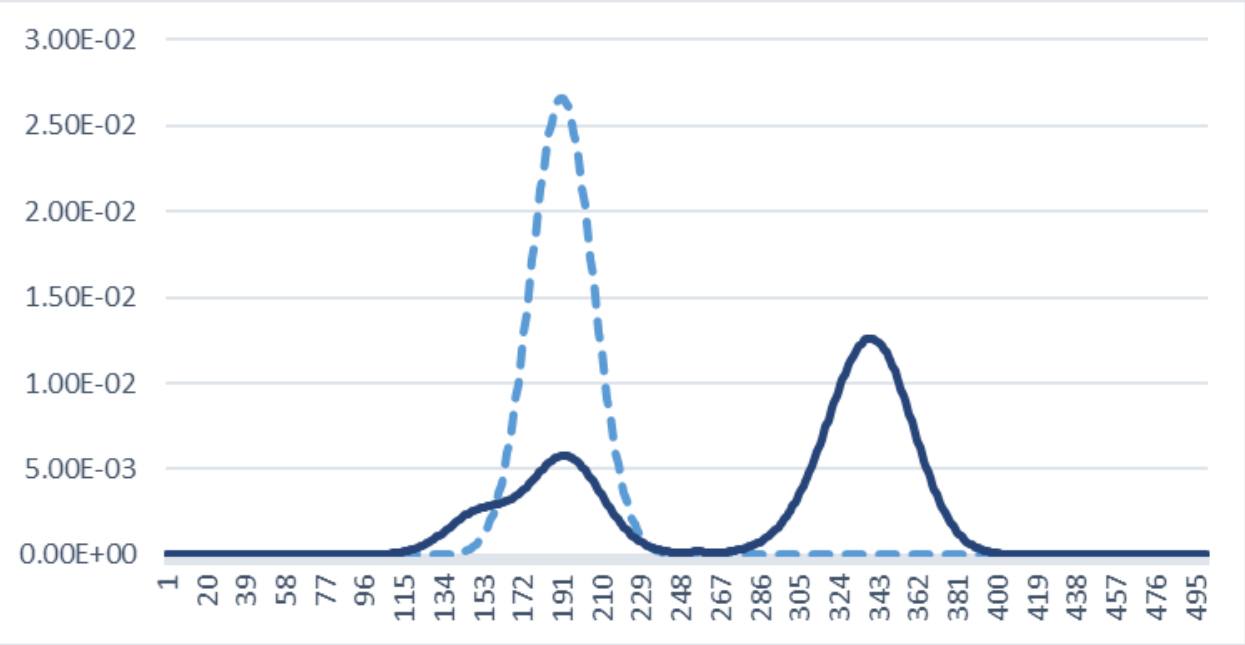

Figure 2: Quantum scattering of a wave packet through a potential barrier of height $U$ and width $W=10 \ell$, with $\ell=1$. The bell-shaped incident wave packet (probability distribution: dashed line) has the average energy $E_{0}$ with $E_{0} / U=\pi^{2} / 8.0, k_{0}=\pi / 6$, and $\sigma=15 \ell$. The reflected and transmitted wave (probability distribution) is shown as the solid line in the figure. The potential locates between the lattice site 251 and 261 .

a value that is close to 0.632 by theoretical calculation

$$
\mathrm{P}_{t h}(\text { transmission })=\int d k \frac{4 \varepsilon_{k}\left(\varepsilon_{k}-U\right)|\Phi(k)|^{2}}{4 \varepsilon_{k}\left(\varepsilon_{k}-U\right)+U^{2} \sin ^{2}(\alpha(k) W)},
$$

where $W$ denotes the width of the square potential, and

$$
\alpha(k)=\sqrt{\frac{2 M\left(\varepsilon_{k}-U\right)}{\hbar^{2}}}
$$

could be either real or imaginary depending on whether $\left(\varepsilon_{k}-U\right)$ is positive or negative. The numerical calculation which is based on the standard central difference equation gives 0.603 for the transmission probability, about 5 percents lower than the theoretical value. The accuracy can be enhanced further to give 0.633 for the transmission probability if $\ell=1 / 3$ is chosen and the space has 1500 lattice sites. The value of $k_{0} \ell$ is reduced from $\pi / 6>0.35$ for $\ell=1$ to $\pi / 18<0.35$ for $\ell=1 / 3$. Smaller value of $k_{0} \ell$ usually leads to the numerical 70 result with higher accuracy as expected. 
Other than the aspect of numerical calculation, the exact discretized Schrödinger equation does present a non-local transport behavior of particles. In the Schrödinger field theory, a spatially localized quantum particle at location $x$ can jump to any location $x+m \ell(m \neq 0)$ with a probability in a short time $\Delta t$

$$
\operatorname{Prob}(x \rightarrow x+m \ell)=\left(\frac{\hbar \Delta t}{M m^{2} \ell^{2}}\right)^{2},
$$

not just jump to its nearest neighboring sites. With the probability, the expected value of $m^{2} \ell^{2}$ is

$$
\left\langle m^{2} \ell^{2}\right\rangle=\left(\frac{\hbar \pi \Delta t}{\sqrt{3} M \ell}\right)^{2} .
$$

It implies that, in a short time interval $\Delta t$, the standard deviation of the jumping distance for the highly localized particle is $\hbar \pi \Delta t /(\sqrt{3} M \ell)$. Thus the standard deviation of the momentum $P$ for the localized particle is $\Delta P=\hbar \pi /(\sqrt{3} \ell)$, and the uncertainty relation holds

$$
(\Delta P) \ell=\frac{\hbar \pi}{\sqrt{3}}>\Delta P \Delta x \geq \frac{\hbar}{2} .
$$

\section{Conclusion}

In this paper we show that the exact discrete analogue of Schrödinger equation can be derived naturally from the Hamiltonian operator that is given in momentum representation after the second quantization of the Schrödinger field theory. By defining the field operators $a_{x}$ and $a_{x}^{\dagger}$ to be the discrete Fourier transforms of $a_{k}$ and $a_{k}^{\dagger}$, the Hamiltonian operators can be transformed into that in position representation. The exact discretized Schrödinger equation is then easily derived from time evolution of quantum states. The position and momentum operators are also constructed in position representation in the paper. The commutator relation between the two operators in discrete space is also derived and found to be different from the conventional commutator relation in continuous space. This results from the fact that both momentum and position eigenkets can be the single-particle quantum states for the Schrödinger field theory in 
discrete space. In the continuous limit $(N \rightarrow \infty, \ell \rightarrow 0)$, the commutator relation in discrete space is shown to go back to the conventional one in continuous space. Though in the paper we assume that the creation and annihilation operator satisfy bosonic quantization relation $\left[a_{k}, a_{k^{\prime}}^{\dagger}\right]=\delta_{k k^{\prime}}$, the results in the previous sections including the exact discrete analogue of Schrödinger equation, and the commutation relation between position and momentum operator, are remained the same with fermionic quantization relation $\left\{a_{k}, a_{k^{\prime}}^{\dagger}\right\}=\delta_{k k^{\prime}}$.

A comparison between the exact discrete analogue of Schrodinger equation and the standard central difference equation is made by numerically studying the transmission probability for a particle passing through a square potential barrier in one dimensional space. In the quantum scattering problem, both discretization schemes give sensible and accurate results as compared to theoretical calculation. Usually it will take more computation time when using exact discretization formula in numerical calculation since it needs one to sum up contributions from the wavefunctions at all spatial sites. On the other hand, the condition $\left|k_{0} \ell\right|<0.35$ must be satisfied if we want to have an accurate numerical result by using the standard central difference equation. Sometimes it means that a smaller lattice spacing $\ell$ and thus a larger number of spatial lattice sites must be chosen in numerical calculation.

Conceptually, the exact discretized Schrödinger equation is more quantumlike than the standard central discretization since it allows particles to jump to remote sites via the hopping interaction terms $a_{x+m \ell}^{\dagger} a_{x}$ in the Hamiltonian operator. The hopping interactions origin from the free Hamiltonian operator and play important roles in particle transport. The theory that leads to the standard central discretization simply does not have such hopping terms except for the jumps to nearest neighboring sites $a_{x \pm \ell}^{\dagger} a_{x}$.

\section{References}

[1] P. G. Kevrekidis, the Discrete Nonlinear Schrodinger Equation, SpringerVerlag, 2009. 
[2] T. B. Boykin, G. Klimeck, The discretized schrdinger equation and simple models for semiconductor quantum wells, Eur. J. Phys. 25 (2004) 503-514. doi:10.1088/0143-0807/25/4/006

[3] V. Tarasov, Exact discretization of schrodinger equation, Phys. Lett. A 380 (2016) 68-75. doi:10.1016/j.physleta.2015.10.039

[4] D. J. Griffiths, Introduction to Quantum mechanics, Pearson Education, Inc., 2005.

[5] V. Tarasov, Exact discrete analogs of canonical commutation and uncertainty relations, Mathematics 4 (2016) 44. doi:10.3390/math4030044.

[6] H. Krenzlin, J. Budczies, K. Kehr, Wave packet tunneling, annalen der

1. Physik 7 (1998) 732-736. doi:10.1002/(SICI) 1521-3889(199812) 7:7/ 8<732: :AID-ANDP732>3.0.CO;2-0.

125 [7] R. M. Dimeo, Wave packet scattering from time-varying potential barriers in one dimension, Am. J. Phys. (2014) 142doi:10.1119/1.4833557. 